\title{
XLV. On some problems connected with the flow of electricity in a plane
}

\section{Oliver J. Lodge B.Sc.}

To cite this article: Oliver J. Lodge B.Sc. (1876) XLV. On some problems connected with the flow of electricity in a plane, Philosophical Magazine Series 5, 1:5, 373-389, DOI: $10.1080 / 14786447608639055$

To link to this article: http://dx.doi.org/10.1080/14786447608639055

曲 Published online: 13 May 2009.

Submit your article to this journal $\sqsubset x$

Џ Article views: 4

Q View related articles $\asymp$ 
Now it has been shown by Boltzmann that if, in such a gas, the number of variables in each molecule be $n$, the total enorgy $\mathrm{T}$ is to the energy of translation $\mathrm{T}_{1}$ as $n$ to 3 , or

We have therefore

$$
\mathrm{T}_{1}=\frac{3 \mathrm{~T}}{n}
$$

and

$$
p d v=\frac{2 \mathrm{~T}}{n} \frac{d v}{v}-\frac{d \mathrm{U}^{\prime}}{d v} d v, \ldots . . . .
$$

$$
\frac{d \mathrm{Q}}{\mathrm{T}}=d \log \mathrm{T}+\frac{2}{n} d \log v-\frac{d}{d \mathrm{~T}} \frac{d \mathrm{U}^{\prime}}{d v} d v+\frac{d \mathrm{U}}{d \mathrm{~T}} d \log \mathrm{T} .
$$

Or, observing that $\mathrm{T}_{1}$ and not $\mathrm{T}$ is here the measure of temperature, we may write

$$
\frac{d \mathrm{Q}}{\mathrm{T}_{1}}=\frac{n}{3} d \log \mathrm{T}_{1}+\frac{2}{3} d \log v-\frac{d}{d \mathrm{~T}_{1}} \frac{d \mathrm{U}^{\prime}}{d v} d v+\frac{d \mathrm{U}}{d \mathrm{~T}_{1}} d \log \mathrm{T}_{1},
$$

the condition necessary to make $\frac{d \mathrm{Q}}{\mathrm{T}}$ or $\frac{d \mathrm{Q}}{d \mathrm{~T}_{1}}$ an exact differential remaining the same as in the case first examined, as expressed in equation (5), except that $\frac{d \mathrm{U}^{\prime}}{d v} d v$ is not the variation of the total potential upon the hypothesis of uniform expansion at any instant, but only such variation of the potential of the forces between the molecules.

XLV. On some Problems connected with the Flow of Electricity in a Plane. By OLIver J. LodGe, B.Sc.*

$7 \mathrm{HE}$ following paper may be regarded as a sort of appendix 1 to a paper by Professor G. C. Foster and myself, entitled "On the Flow of Electricity in a uniform plane Conducting Surface," and published in the Philosophical Magazine for May, June, and December 1875 ([IV.] vol. xlix. pp. 385-400 and 453-471, and vol. l. pp. 475-489), in which the known laws of the flow of electricity in an unlimited plane were deduced from the fundamental idea that the effects of any number of poles could be obtained by simple summation of the effects due to each pole separately, without using any mathematics higher than elementary algebra. Several questions arose in the course of the paper which it was not thought advisable to consider then, but some of which seemed to deserve a separate treat-

* Read before the Physical Society on the 26th of February and the 25th of March, 1876. Communicated by the Society. 
ment; these are the subjects I propose to develop in the present communication.

$\S 1$. Imagine a plane sheet, some portions having infinite conducting-power, other portions consisting of ordinary matter, and again other portions possessing infinite resisting-power. The whole extent of any of the infinitely conducting regions will have a uniform potential ; and hence the bounding line between any such region and the ordinary matter of the sheet must be an equipotential line. Further, if one defines a line of flow or stream-line as a line across which no electricity passes, the boundary line between the ordinary matter of the sheet and an infinitely resisting region must be a line of flow, for no electricity can possibly get across it. Such a sheet will thereforo contain a certain number of flow-lines and a certain number of equipotential lines. Moreover let the potential of any one of the conducting regions be maintained higher than that of some other one, then in general a uniform flow of electricity will take place throughout the whole of the ordinary matter of the sheet, which will therefore contain additional stream-lines and equipotential lines, whose arrangement will depend on the situations and potentials of the various non-material regions.

Some general properties of the equipotential and streamlines were pointed out in $\S 35$ of the paper referred to. One of these properties is, of course, that the lines of one system cut the lines of the other orthogonally; a more general statement is the following. If a flow-line passes $n$ times through a given point of the sheet, it will form at that point an equiangular pencil whose $2 n$ rays meet each other at an angle $\frac{\pi}{n} ;$ and if no pole exists at the meeting-point it will be a point of no flow, and an equipotential line will also pass $n$ times through the same point, making an equiangular pencil the same in all respects as the former, but turned through an angle of $\frac{\pi}{2 n}$, so that its rays bisect the angles of the first pencil. The ordinary orthogonal section is the special case of this when $n=1$. The special case $n=\infty$ is obtained if, in the sheet imagined above, one of the conducting regions touches one of the resisting regions along a line; when this happens, the line separating the two may be regarded indifferently as a stream-line or as an equipotential line.

There are then two ways of making any given line a streamline-viz. either by arranging the non-conducting regions so that the line shall bound one of them, or else by arranging the conducting regions and their potentials in such a way that none of the flow of electricity shall take place across any por- 
tion of the given line. Similarly any line may be made an equipotential line, either by making it bound a region of infinite conductivity, or else by adjusting the potentials and positions of these same regions so that no part of the flow shall take place along the given line.

Such a sheet as we have imagined can be approximated to by a piece of tinfoil cut to any shape, with any number of holes in it, and having on its surface blocks of clean copper which are connected with a thermo-pile or other means of maintaining constant difference of potential.

$\S 2$. The conditions of flow in such a sheet, however, are in general unknown. But if infinitely resisting regions are absent, and if the infinitely conducting regions are reduced to mere points of infinite potential, we get the case of an unlimited sheet containing point poles; and in this all the conditions of the flow are completely known for every point of the sheet. Thus the potential at any point distant $r_{1}, r_{2} \ldots$ from the poles which emit quantities of electricity $q_{1}, q_{2} \ldots$ in unit time, and which tend to produce potentials $\phi_{1}, \phi_{2} \ldots$ at unit distance from themselves, is ( $\$ 33$ of the former paper)

$$
\mathrm{V}=\Sigma \phi-\frac{1}{2 \pi \kappa \delta} \Sigma q \log r, . . . . .
$$

where $\delta$ is the thickness and $\kappa$ the specific conductivity of the sheet; and from this expression stream-line equations and resistance expressions follow.

So, then, the problem of finding the flow conditions in any bounded sheet containing point poles will be solved if we can imitate the electrical boundary conditions in an unlimited sheet by introducing extra point poles into it. These additional poles are called images in the boundary, because they produce the same effect in the given portion of the sheet as the boundaries themselves produced-just as the illumination inside a mirrorwalled room containing candles would be imitated in unlimited space by placing extra candles at all the points occupied by the images of the original candles in the mirrors.

\section{Images in rectilinear boundaries.}

$\S 3$. Now let it be required to cause a given straight line in an infinite sheet of tinfoil to be a stream-line. All that is necessary is to arrange all the blocks of copper in the sheet symmetrically with respect to this line; that is to say, they must either be placed symmetrically upon the line, or they must be placed in pairs one on each side of it and at equal distances from it. It is usual to express this fact in the inverse way thus :-The image of a pole in a straight flow-line is a pole of 
the same size, shape, and strength, and situated at an equal distance on the other side of the line so as to be opposite to the first pole.

Again, in order to make a given straight line into an equipotential line, the blocks must be arranged skew-symmetrically with respect to the line - that is to say, in pairs one on each side of it and at equal distances from it, but the sign of one of the constituents of each pair must be opposite to that of the other. This fact is expressed thus :-The image of a pole in a straight equipotential line is a pole of the same size, shape, and numerical strength, but of opposite sign, and situated at an equal distance on the other side of the line.

Hence, if we have given a sheet of tinfoil bounded by a straight edge ( $i$. e. by a straight flow-line) and with any distribution of poles in it whatever, and we wish to find the distribution of poles which would make that straight line a flowline in the unlimited sheet, we have only to regard the line as a plane mirror whose silvered face is turned towards the sheet, and the optical images of the several poles will give the distribution required. The distribution of poles will be obtained in the same way if the boundary is a straight band of copper ( $i . e$. an equipotential line); but in this case the sign of every image must be reversed.

$\S 4$. Now take a sheet bounded by two straight flow-lines intersecting one another at any angle $\theta$ : every pole in such a sheet will be reflected backwards and forwards between the two edges according to optical laws; and hence it should have a finite series of images lying on a circle and agreeing with those in the kaleidoscope, the reflection coming to an end as soon as a couple of images fall in the space which is behind both mirrors. But here arises a difficulty. When $\theta$ is a submultiple of $\pi$, the last couple of images coincide and the whole set is symmetrical with respect to both the straight lines, as is required. But if $\theta$ is not a submultiple of $\pi$, all that happens in the case of the kaleidoscope is that the last two images separate, the apparent number of images being simply increased by 1 . But now the images are not arranged symmetrically with respect to both the straight lines, and hence they cannot both be flow-lines. In order to obtain symmetry, it is necessary to continue the reflecting process as if the back of the mirrors were silvered as well as the front; and in that case, of course, the number of images becomes infinite. But then some of these infinite images will fall inside the angle $\theta$ (that is, will come into the sheet itself), which will be inconsistent with the given distribution of poles in the sheet. Hence it appears to be impossible for the uniform infinite sheet to contain two 
straight flow-lines which enclose any angle but a submultiple of $\pi$, unless the poles are put infinitely close together, forming a complete circular ring whose centre is at the intersection of the two lines; and in this case every straight line drawn in the sheet from this centre is a flow-line.

Another way of expressing the facts is to say that both sides of every flow-line are to be regarded as mirrors, and its images as always infinite in number though often coincident with one another, and that the problem of reducing the bounded case to that of the infinite sheet becomes impossible whenever the images reflected back into the sheet itself do not coincide both in sign and position with the images already there. Similar statements are true of the images in a pair of intersecting equipotential lines.

Another distinction between optical and electrical reflection is, that when two optical images coincide their light-emitting powers are added, but, in the electrical case, coincidence of one image with another does not increase its strength. These differences appear to forbid our imagining that there is any physical meaning in electrical reflection at boundaries analogous to the physical reflection of the waves of light in a mirror.

$\$ 5$. Consider now the images of a point inside a polygon of $n$ sides. We have seen that, without silvering both faces of the boundaries and thereby introducing an unconscionable number of images, some of them real, we can only treat angles that are submultiples of $\pi$ by the method of images. Hence obtuse angles less than $\pi$ are not allowable. 'This condition at once excludes all polygons of more than four sides; for as the largest interior angle of a polygon of $n$ sides cannot be less than $\frac{n-2}{n} \pi$, and as it must be less than or equal to $\frac{\pi}{2}$, it is necessary that $n$ shall be equal to or less than 4 . And if $n=4$, the polygon must be equiangular, that is, must be a rectangle. If $n=3$, the triangles whose angles are submultiples of $\pi$ are the equiangular, the isosceles right angled, and the one whose angles are $90^{\circ}$, $60^{\circ}$, and $30^{\circ}$. The pseudo-triangle whose angles are $90^{\circ}, 90^{\circ}$, and $0^{\circ}$ is also a possible case, and is treated below as a special case of the circular sector.

I have not mentioned the case when $\pi$ itself is one of the angles of a polygon, because this merely reduces the polygon to one of $n-1$ sides; but there is the case of the equiangular polygon with all its angles $\pi$, viz. the circle, about which a little shall be said later.

The images of a point in a rectangle occur in groups of four surrounding the vertices of rectangles whose linear dimensions 
are twice those of the original one, and which cover all the plane.

The images of a point in an equilateral triangle occur in groups of six surrounding the vertices of triangles $\sqrt{3}$ times the linear dimensions of the original one, and covering all the plane.

\section{Application of images to certain cases of bounded plates.}

$\S 6$. Now although the potential of any point is really determined by (A), 2, as soon as we know the positions of all the images in the sheet, the expression is not in a practicable form; and it would not in the majority of cases be readily. possible to bring it to such a form. The way to proceed in such cases is to consider what is required of the potential, and then to write down a function of $x+i y$,

$$
\mathrm{W} \equiv \mathrm{V}+\mathrm{U} i
$$

such that the real part satisfies all the conditions required of the potential function, viz. to remain together with its derivatives continuous and finite all over the sheet except at the poles and their images, where it is to become infinite like $q \log r$, to fulfil certain given conditions at the boundary, and to satisfy the equation

$$
\frac{d^{2} \mathrm{~V}}{d x^{2}}+\frac{d^{2} \mathrm{~V}}{d y^{2}}=0
$$

This being done, $\mathrm{V}=$ const. will represent the equipotential lines, and $\mathrm{U}=$ const. the stream-lines, and the components $\frac{d \mathrm{~V}}{d x}, \frac{d \mathrm{~V}}{d y}$ of the flow at any point can be obtained, In this way Dr. E. Jochmann* has expressed all the conditions of the flow in a rectangle, bounded either by stream-lines or by equipotential lines, or by both.

\$7. But though the potential expression (A) is often of impracticable form, the expression for the resistance of a conductor, which follows from it, is simpler, and admits of being reduced to a useful form with much ease in many cases. Expressions so obtained may serve to verify the results of more abstruse and general processes ; but the principal reason for entering into the subject is that the expressions themselves and several of the continued products which lead to them seem to be of some little mathematical interest.

* "Ueber einige Aufgaben, welche die Theorie des logarithmischen Potentials und den Durchgang eines constanten elektrischen Stroms durch eine Ebene betreffen," von Dr. E. Jochmann in Berlin. Schlömilch, Zeitschrift fiir Mathematik, 1865, p. 48. 


\section{Resistance of some rectilinear figures to the current flowing} between two small circular electrodes.

$\S 8$. Let us first remind ourselves how the general resistanceexpression is obtained. Given an infinite sheet with any number $k$ of equal sources $A_{1}, A_{2}, \ldots A_{k}$ and the same number of equal sinks $\mathrm{B}_{1}, \mathrm{~B}_{2}, \ldots \mathrm{B}_{k}$ in it, the resistance between the two equipotential lines which pass through any two fixed points $P$ and $Q$ is given by $\mathrm{Ohm}$ 's law as

$$
\mathrm{R}_{\mathrm{PQ}}=\frac{\nabla_{\mathrm{P}}-\mathrm{V}_{\mathrm{Q}}}{k q},
$$

where $V_{P}$ stands for the actual potential at the point $P$, and $k q$ stands for the whole strength of current flowing in the sheet-that is to say, for the quantity of electricity emitted by each source per second, multiplied by the number of sources. Now (A) gives as the potential at any point $P$

hence

$$
\mathrm{V}_{\mathrm{P}}=-\frac{q}{2 \pi \kappa \delta} \log \frac{\mathrm{A}_{1} \mathrm{P} \cdot \mathrm{A}_{2} \mathrm{P} \ldots,}{\mathrm{B}_{1} \mathrm{P} \cdot \mathrm{B}_{2} \mathrm{P} \ldots}, . . . . .
$$

$$
R_{P Q}=\frac{1}{2 k \pi \cdot \kappa \delta} \log \left\{\frac{A_{1} Q \cdot A_{2} Q \ldots}{B_{1} Q \cdot B_{2} Q \ldots} \cdot \frac{B_{1} P \cdot B_{2} P \ldots}{A_{1} P \cdot A_{2} P \ldots}\right\} .
$$

And these expressions are perfectly general; for unequal poles may be regarded as aggregations of equal ones, and the number of sinks must always be equal to the number of sources, although some of them may often be at infinity.

Now we know that the equipotential lines of high potential break up into $k$ portions which ultimately become little circles, one surrounding each source. Similarly the lines of low potential surround the sinks. Take the point $\mathbf{P}$ on the circumference of one of these little circles (radius $\rho_{1}$ ), that surrounding the source A say (omitting its suffix); and take the point $Q$ on a circle (radius $\rho_{2}$ ) surrounding the sink $B$. Then the resistance between the two small circular electrodes $A$ and $B$ is $k$ times as much as that offered by the sheet to the $2 k$ poles, or

$$
\mathrm{R}_{\mathrm{AB}}=\frac{1}{2 \pi i \delta} \log \left\{\frac{\mathrm{AB} \cdot \mathrm{A}_{1} \mathrm{~B} \cdot \mathrm{A}_{2} \mathrm{~B} \ldots}{\rho_{1} \cdot \mathrm{A}_{1} \mathrm{~A} \cdot \mathrm{A}_{2} \mathrm{~A} \ldots} \cdot \frac{\mathrm{BA} \cdot \mathrm{B}_{1} \mathrm{~A} \cdot \mathrm{B}_{2} \mathrm{~A} \ldots}{\rho_{2} \cdot \overline{\mathrm{B}_{1}} \bar{B} \cdot \mathrm{B}_{2} \overline{\mathrm{B}} \ldots}\right\},(\alpha)
$$

where the higher powers of $\rho_{1}$ and $\rho_{2}$ are neglected. Since they are both small there is no necessity to distinguish them, and $\rho^{2}$ may be written for their product. Moreover in very many cases the sources and sinks will be similarly arranged; so that the two fractions which are multiplied together inside the brackets of $(\alpha)$ are equal to one another, and we may then use 
the simplified expression

$$
R_{A B}=\frac{1}{\pi \kappa \delta} \log \left(\frac{A B \cdot A_{1} B \cdot A_{2} B \cdots}{\rho \cdot A_{1} A \cdot A_{2} A \cdots}\right) . .
$$

$\S 9$. Before proceeding to apply the image theory and this expression to the general case of a two-sided figure, it will be well to consider a special case or two,-and first the very simple case of a parallel-sided strip of breadth $s$, with the two poles, A and B, one on each side of the strip and opposite to one another (fig. 1).

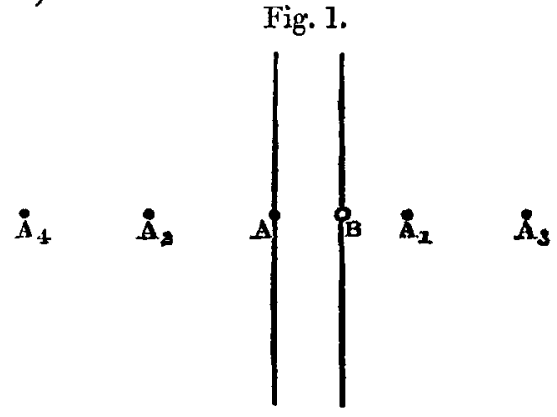

A will be reflected in the opposite side, its image $A_{1}$ being at a distance $s$ from that side and from $B$; then $A_{1}$ will be reflected, its image $A_{2}$ being $2 s$ distant from $A$ and $3 s$ from $B$; then $A_{2}$ will be reflected, and so on. The images of $B$ will evidently be similarly arranged to those of A mutatis mutandis; so we may use the second of the resistance-formulæ

$$
\mathrm{R}=\frac{2}{\pi \kappa \delta} \log \frac{\mathrm{AB} \cdot \mathrm{A}_{1} \mathrm{~B} \ldots}{\rho \cdot \mathrm{A}_{1} \mathrm{~A} \ldots}
$$

doubling it because the poles are only halfway on the sheet (see below, \$22). Now

$$
\begin{array}{ll}
\mathrm{AB}=\mathrm{A}_{1} \mathrm{~B}=s, & \mathrm{~A}_{1} \mathrm{~A}=\mathrm{A}_{2} \mathrm{~A}=2 s, \\
\mathrm{~A}_{2} \mathrm{~B}=\mathrm{A}_{3} \mathrm{~B}=3 s, & \mathrm{~A}_{3} \mathrm{~A}=\mathrm{A}_{4} \mathrm{~A}=4 s, \\
\mathrm{~A}_{4} \mathrm{~B}=\mathrm{A}_{5} \mathrm{~B}=5 s, & . . .+. .
\end{array}
$$

Hence the above product becomes

which equals $\frac{s}{\rho} \cdot \frac{2}{\pi}$.

$$
\frac{s}{\rho} \cdot \frac{1.3 .3 .5 .5 .7 \ldots}{2.2 .4 .4 .6 .6 \ldots}
$$

Therefore

$$
\mathrm{R}=\frac{2}{\pi \kappa \delta} \log \frac{2 s}{\pi \rho} . \quad \cdot \cdot \cdot \cdot \cdot
$$


$\S 10$. One more special case of the infinite strip I will take, because a constantly occurring product occurs here in its simplest form. Let the two poles be both on the same side of the strip and at a distance $c$ from one another. The images of $A$ are just as before, and the product $\frac{A B}{\rho} \cdot \frac{A_{1} B}{A_{1} A} \cdot \frac{A_{2} B}{A_{2} A} \ldots$ equals

$$
\begin{aligned}
& \left\{\frac{c^{2}}{\rho^{2}} \cdot \frac{2^{2} s^{2}+c^{2}}{2^{2} s^{2}} \cdot \frac{2^{2} s^{2}+c^{2}}{2^{2} s^{2}} \cdot \frac{4^{2} s^{2}+c^{2}}{4^{2} s^{2}} \cdot \frac{4^{2} s^{2}+c^{2}}{4^{2} s^{2}} \ldots\right\}^{\frac{1}{2}} \\
& =\frac{c}{\rho}\left(1+\frac{c^{2}}{2^{2} s^{2}}\right)\left(1+\frac{c^{2}}{4^{2} s^{2}}\right)\left(1+\frac{c^{2}}{6^{2} s^{2}}\right) \ldots . \\
& =\frac{c}{\rho} \cdot \frac{\sinh \frac{\pi c}{2 s}}{\frac{\pi c}{2 s}} * .
\end{aligned}
$$

Therefore

$$
\mathrm{R}=\frac{2}{\pi \kappa \delta} \log \left(\frac{2 s}{\pi \rho} \sinh \frac{\pi c}{2 s}\right) . \quad . \quad . \quad . \quad . \quad .
$$

Similarly, if we had taken the poles one on each side of the strip, but, instead of opposite each other as at first, at a distance $c$ from one another measured parallel to the sides of the strip, we should have obtained

$$
\mathrm{R}=\frac{2}{\pi \kappa \delta} \log \left(\frac{2 s}{\pi \rho} \cosh \frac{\pi c}{2 s}\right) . . . . . . .
$$

If $s$ be made infinite, (2) becomes $\frac{2}{\pi \kappa \delta} \log \frac{c}{\rho}$, which is the correct expression for the case of two poles on the edge of half an infinite sheet. If $s$ is small, (2) and (3) become equal;

* Here and elsewhere sinh $x$ may be considered merely an abbreviation for $\frac{e^{x}-e^{-x}}{2}$, or as equal to $\frac{1}{i} \sin i x$. Similarly

and

$$
\cosh x=\frac{e^{x}+e^{-x}}{2} \text {, or }=\cos i x,
$$

$$
\tanh x=\frac{e^{x}-e^{-x}}{e^{x}+e^{-x}}=\frac{1}{i} \tan i x,
$$

where $i=\sqrt{-1}$. Il is useful to remember that

$$
\begin{array}{ll}
\text { that } & \cosh ^{2} x-\sinh ^{2} x=1, \\
\text { that } & \sinh 0=\tanh 0=\sin 0=0, \\
\text { and that } & \cosh 0=\tanh \infty=1, \\
& \sinh \infty=\cosh \infty=\frac{1}{9} e^{\infty}
\end{array}
$$


and if $s=\rho$, they reduce to $\mathrm{R}=\frac{c}{\kappa \rho \delta}$ (neglecting the small term $-\frac{2}{\pi \kappa \delta} \log \pi$ ), which is right for the resistance of a wire or thin strip of sectional area $\rho \delta$ and length $c$. The presence of the term neglected in comparison with a term which has $\rho$ in its denominator, can be accounted for by remembering that the electrodes are not exactly straight bars at a distance $c$, butquadrants of small circles whose centres are distant $c$ from one another.

$\S 11$. We may also consider one special case of the infinite "wedge" or irregular two-sided polygon. Let the two poles be one on each side of the wedge at the same distance $r$ from its angle $O$, and call this angle $\theta=\frac{\pi}{n}$ ( $n$ integer). The images of $A$ will lie on a circle with centre $O$ (fig. 2), as in the kaleidoscope. Fig. 2.

Then

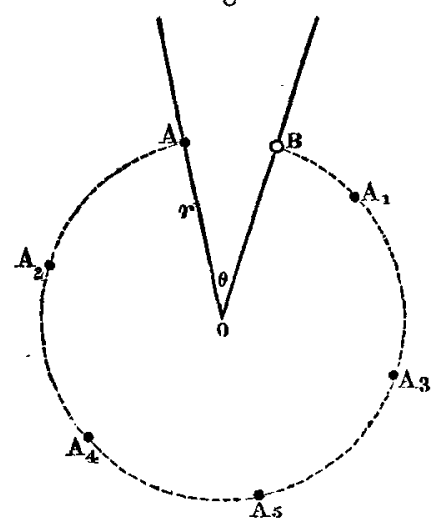

$$
\begin{array}{ll}
\mathrm{A} B=\mathrm{A}_{1} \mathrm{~B}=2 r \sin \frac{\theta}{2}, & \mathrm{~A}_{1} \mathrm{~A}=\mathrm{A}_{2} \mathrm{~A}=2 r \sin \frac{2 \theta}{2}, \\
\mathrm{~A}_{2} \mathrm{~B}=\mathrm{A}_{3} \mathrm{~B}=2 r \sin \frac{3 \theta}{2}, & \mathrm{~A}_{3} \mathrm{~A}=\mathrm{A}_{4} \mathrm{~A}=2 r \sin \frac{4 \theta}{2},
\end{array}
$$

so the product which occurs in $(\beta)$ equals

$$
\frac{2 r}{\rho} \cdot \frac{\sin ^{2} \frac{\theta}{2} \sin ^{2} \frac{3 \theta}{2} \sin ^{2} \frac{5 \theta}{2} \cdots}{\sin ^{2} \frac{2 \theta}{2} \sin ^{2} \frac{4 \theta}{2} \sin ^{2} \frac{6 \theta}{2} \ldots}\left\{\begin{array}{l}
\frac{\sin ^{2} \frac{n-1}{2} \theta}{\sin ^{2} \frac{n}{2} \theta} \sin \frac{n \theta}{2} \text { when } n \text { is even } \\
\frac{\sin ^{2} \frac{n-2}{2} \theta}{\sin ^{2} \frac{n-1}{2} \theta} \sin \frac{n \theta}{2} \text { when } n \text { is odd. }
\end{array}\right.
$$


with the Flow of Electricity in a Plane.

But we know from Trigonometry that

and that

$$
\sin \frac{\pi}{2 n} \sin \frac{3 \pi}{2 n} \ldots\left\{\begin{array}{l}
\sin \frac{n-1}{2 n} \pi \\
\sin \frac{n-2}{2 n} \pi
\end{array}=2^{\frac{2}{n-1}},\right.
$$

$$
\sin \frac{2 \pi}{2 n} \sin \frac{4 \pi}{2 n} \ldots\left\{\begin{array}{l}
\sin \frac{n-2 \pi}{2 n} \pi \\
\sin \frac{n-1}{2 n} \pi
\end{array}=2^{\frac{2}{n-1}} \sqrt{n},\right.
$$

the upper termination to be taken when $n$ is even, the lower when $n$ is odd. Hence the above product equals

and

$$
\frac{2 r}{\rho} \cdot \frac{1}{n}
$$

$$
\mathrm{R}=\frac{2}{\pi \kappa \delta} \log \frac{2 r \theta}{\pi \rho} \text { or }=\frac{2}{\pi \kappa \delta} \log \frac{2 s}{\pi \rho}, . . .
$$

if one writes $s$ for the are subtending $\theta$ of the circle whose radius is $r$.

Notice here the close resemblance to (1), becoming identity when $r=\infty$, and also the sort of circular analogy to Wallis's form of $\frac{\pi}{2}$ which one gets by taking arcual instead of linear distances in fig. 2 for the product of $(\beta)$.

The circle on which all the poles lie is a flow-line by symmetry; and hence the resistance of the sector A O B is twice that of the wedge.

\section{General resistance of a "Wedge," or two-sided polygon, poles any where.}

$\$ 12$. If we obtain an expression for the case of two poles $A$ and $B$, it will be little more than a matter of writing to extend it to any number. Let $O X$ and $O Y$ be the two sides of the wedge, the angle $\mathrm{YOX}$ being $\theta=\frac{\pi}{n}$. Call the distance $\mathrm{OA}$, $r_{1}$; and the angle $\mathrm{A} \mathrm{OX}, \alpha(\mathrm{fig} .3)$; and let $\left(r_{2}, \beta\right)$ denote the point $B$. The images of $A$ will lie in pairs on a circle of radius $r_{1}$, those of $B$ on a circle of radius $r_{2}$; and to find the value of the products which occur in the general expression $(\alpha)$ we may make use of Cotes's (or Demoivre's) property of the circle, which says that, if $m$ points equisect the circumference of a circle of radius $r_{1}$, the product of their distances from anFig. 3.

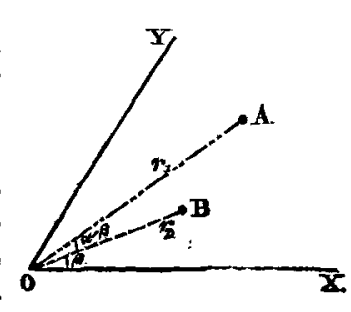


other point, distant $r_{2}$ from the centre of the circle and $\checkmark\left(r_{1}^{2}-2 r_{1} r_{2} \cos \phi+r_{2}^{2}\right)$ from the nearest of the $n$ points, will be equal to

$$
\sqrt{ }\left(r_{1}^{2 m}-2 r_{1}^{m} r_{2}^{m} \cos m \phi+r_{2}^{2 m}\right) . \quad \text {. . . . }
$$

To apply this theorem to the present case we must consider the images of $A$ in two sets:- one set including $A$ itself and all its images whose angular distance from it is some multiple of $2 \theta$; the other set containingall the remaining images, which will alternate with those of the first set, and will like them divide the circle into $n$ equal parts. The product of all the distances of the first set from the point $\mathrm{B}$ will be given by (C) if we put $m=n=\frac{\pi}{\theta}$ and $\phi=\alpha-\beta$; the product for the second set will only differ in having $\phi=\alpha+\beta$. From the symmetry of the expression, it follows that the product of the distances of the images of $B$ from the point $A$ will be the same as that of the images of A from the point B. Hence the numerator of $(\alpha)$ is determined. As for the denominator, the product of the distances of the second set of images of $A$ from the point $A$ is

$$
2 r_{1}^{n} \sin n \alpha .
$$

The product of the first set appears to be zero, since A itself is one of the set; but remembering that the point to which distances are properly measured is not the actual pole $\mathrm{A}$ but a point on the circumference of its small electrode $(\$ 8)$, we see that the product becomes

$$
r_{1}^{n}-\left(r_{1}-\rho\right)^{n}=n r_{1}^{n-1} \rho,
$$

neglecting high powers of $\rho$. So the whole product of the A's from $A$ is $2 n \rho r_{1}^{2 n-1} \sin n \alpha$; similarly the product of the B's from $B$ will be

$$
2 n \rho r_{2}^{2 n-1} \sin n \beta \text {. }
$$

Hence the resistance-expression ( $\alpha$ ) reduces to

$$
\begin{aligned}
& \Re=\frac{1}{2 \pi \kappa \delta} \log \\
& \frac{\left(r^{2 n}-2_{1}^{n} r_{2}^{n} r \cos n(\alpha-\beta)+r_{2}^{2 n}\right)\left(r^{2 n}-2 r_{1}^{n} r_{2}^{n} \cos n(\alpha+\beta)+r_{2}^{2 n}\right)}{4 n^{2} \rho^{2} r_{1}^{2 n-1} r_{2}^{2 n-1} \sin n \alpha \cdot \sin n \beta},
\end{aligned}
$$

or, as it is often more convenient to write it,

$=\frac{1}{2 \pi \kappa \delta} \log$

$$
\left\{\frac{r_{1} r_{2}}{\rho^{2}} \cdot \frac{\left.\left.\left.\left.\left(\frac{r_{1}}{r_{2}}\right]^{n}+\frac{r_{2}}{r_{1}}\right]^{n}-2 \cos n \overline{\alpha-\beta}\right)\left(\frac{r_{1}}{r_{2}}\right]^{n}+\frac{r_{2}}{r_{1}}\right]^{n}-2 \cos n \overline{\alpha+\beta}\right)}{4 n^{2} \sin n \alpha \cdot \sin n \beta}\right.
$$


\$13. A few special cases of this may be recorded.

When $r_{1}=r_{2}$, it becomes

$$
\frac{1}{\pi \kappa \delta} \log \left(\frac{r}{\rho} \cdot \frac{\cos n \alpha-\cos n \beta}{n \sqrt{ }(\sin n \alpha \cdot \sin n \beta}\right) . \quad \text {. . . }
$$

If at the same time $\alpha$ be made zero and $\beta=\theta$, we ought to get (4); but we must remember that $\alpha$ is not actually to vanish, it is to become very small and equal to $\frac{\rho}{2 r}$, which is a sort of compromise between $\frac{\rho}{r}$, the greatest value it could vanish with, and $\frac{0}{r}$, the least. So putting

(6) becomes

$$
\begin{gathered}
\alpha=\frac{\rho}{2 r}, \quad \beta=\theta-\frac{\rho}{2 r}, \\
\frac{1}{\pi \kappa \delta} \log \left(\frac{v}{n \rho} \cdot \frac{2}{\frac{n \rho}{2 r}}\right)=\frac{2}{\pi \kappa \delta} \log \frac{2 r}{n \rho},
\end{gathered}
$$

which agrees with (4).

Going back to $r_{1}$ and $r_{2}$ unequal, let us put both poles on OX-that is, write

(5) will become

$$
\alpha=0=\frac{\rho}{2 r_{1}} \text { and } \beta=0=\frac{\rho}{2 r_{2}} \text {, }
$$

$$
\frac{1}{\pi \kappa \delta} \log \left(\frac{r_{1}^{n}-r_{2}^{n}}{n^{2} \rho^{2} r_{1}^{n-1} r_{2}^{n-1}}\right)^{2} ; \text {. . . . . }
$$

and this reduces to (2) when $r$ is made infinite.

Similarly if the poles were placed one on $O X$ and the other on $\mathrm{OY}$, we should get

$$
\frac{1}{\pi \kappa \delta} \log \frac{\left(r_{1}^{n}+r_{2}^{n}\right)^{2}}{n^{2} \rho^{2} r_{1}^{n-1} r_{2}^{n-1}} ; . . . . . .
$$

and this reduces to (3) when $r_{1}=r_{2}+c=\infty$, and $\theta=\frac{s}{r}=0$.

One more case is worth mentioning, viz. when one of the poles, say $B$, is on the angle of the wedge at $O$. To give this, $r_{2}=\rho$ and ( $\beta$ being any thing between 0 and $\theta, n \beta$ will be any thing between 0 and $\pi$, say $\frac{\pi}{2}$ ) $\sin n \beta=1$, so

$$
\Re=\frac{1}{2 \pi \kappa \delta} \log \left(\frac{v_{1}^{2 n+1}}{\rho^{2 n+1}} \cdot \frac{1}{2 n \sin n \alpha}\right) . \quad \text {. }
$$

Phil. Mag. S. 5. Vol. 1. No. 5. May 1876. 
If at the same time $A$ is on $O X$ or $O Y$, this becomes

$$
\frac{n+1}{\pi \kappa \delta} \log \left\{\frac{r_{1}}{\rho} \cdot\left(\frac{\theta}{\pi}\right)^{\frac{1}{n+1}}\right\} \text {. }
$$

A direct application of Cotes's property and equation (B) to this last case gives us as the perfectly accurate expression

$$
\frac{n}{\pi \kappa \delta} \log \left\{\frac{p_{1}-\rho_{1}}{\rho_{2}}\left(\frac{r_{1}^{n}-\rho_{2}^{n}}{r_{1}^{n}-\left(r_{1}-\rho_{1}\right)^{n}}\right)^{\frac{1}{n}}\right\}, \quad \text {. . }
$$

whatever be the size of the electrodes, provided that they be taken of such shape as to fit the equipotential lines passing through the two points $\mathrm{P}$ and $\mathrm{Q}$ to which distances are measured. Both these points are on the line $A B$, one of them distant $\rho_{1}$ from $A$, the other $\rho_{2}$ from $B$. This expression will reduce to $(10)^{\prime}$ when $\rho_{1}$ and $\rho_{2}$ are regarded as small quantities of the first order.

$\S 14$. Althongh all these expressions are proved only when $\theta$ is a submultiple of $\pi$, yet by continuity they would seem likely to hold true always. If so, we have expressed the resistance of a Riemann's surface of any number of leaves, but all connected at one branch-point $O$.

General resistance of a "Strip," or regular two-sided polygon poles anywhere. Fig. 4 .

$\S 15$ : The strip being a special case of the wedge, its resistance will follow directly from $(5)^{*}$ by making $\theta$ vanish. We must substitute

$$
s=r_{1} \theta, \quad a=r_{1} \alpha, \quad b=r_{2} \beta, \quad c=r_{1}-r_{2},
$$

and then make $r_{1}=r_{2}+c$ infinite, keeping $c, \alpha$, $b, s$ all finite (fig. 4). The substitutions give us

$$
\alpha=\frac{a}{s} \theta, \quad \beta=\frac{b}{s} \frac{r_{1}}{r_{2}} \theta, \quad n=\frac{\pi}{\theta}=\frac{\pi r_{1}}{s} ;
$$

so the resistance $(5)^{\prime}$ becomes

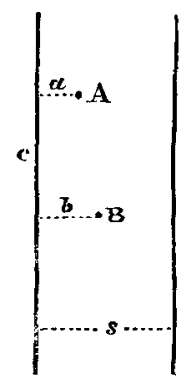

$$
\begin{aligned}
& \Re=\frac{1}{2 \pi \kappa \delta} \log \\
& \left\{\frac{s^{2}}{\pi^{2} \rho^{2}} \cdot \frac{\left(\cosh \frac{c}{s} \pi-\cos \frac{a-b}{s} \pi\right)\left(\cosh \frac{c}{s} \pi-\cos \frac{a+b}{s} \pi\right)}{\sin \frac{a \pi}{s} \sin \frac{b \pi !}{s}}\right\} .
\end{aligned}
$$

* It might be objected that when $n$ is made large, higher powers of $\rho$ in the expansion of $(r-\rho)^{n}$ may not be neglected, as was done in the process of obtaining (5). This would be true if the size of $n$ were merely due to the shutting up of $\theta$ with a constant $r$; but in our present case $r$ is also large, and the expansion is in descending powers of $r$. 
$\$ 16$. For the case when both poles are at the same distance from the sides of the strip, or $a=b$, this becomes

$$
\frac{1}{\pi \kappa \delta} \log \left\{\frac{2 s}{\pi \rho} \sinh \frac{\pi c}{2 s}\left(\frac{\cosh \frac{c \pi}{s}-\cos \frac{2 a \pi}{s}}{1-\cos \frac{2 a \pi}{s}}\right)^{\frac{1}{2}}\right\} ; .
$$

and if they are also both in the middle of the strip, it simplifies to

$$
\frac{1}{\pi \kappa \delta} \log \left(\frac{s}{\pi \rho} \sinh \frac{\pi c}{s}\right)
$$

which is just half (2), as it ought to be. I write out this particularly, because this case has been already studied by Stefan, and experimentally verified by von Obermayr (Wien. Akad. Ber. 1869, vol. lx. part 2. p. 245). Stefan, it seems, wrote the expression he obtained thus (merely altering his letters),

$$
\frac{1}{\pi \kappa \delta} \log \frac{s}{2 \pi \rho}+\frac{1}{\pi \kappa \delta} \log \left(e^{\frac{\pi c}{s}}-e^{-\frac{\pi c}{s}}\right)
$$

and this is identical with $(2)^{\prime}$.

$\S 17$. It seems as if expressions like the above might possibly be employed in obtaining the values of certain continued products. Thus to take (12) as an example.

The direct geometrical product for this case, occurring in $(\beta)$, is the root of

$\frac{c^{2}}{\rho^{2}} \cdot 2 a \cdot \underline{2 s-2 a} \cdot \underline{2 s} \cdot \underline{2 s} \cdot \underline{2 s+2 a} \cdot \underline{4 s-2 a} \cdot \underline{4 s} \cdot \underline{4 s} \cdot \underline{4 s+2 a} \cdot \underline{6 s-2 a} \cdot \ldots$, where $k s \pm 2 a$ is an abbreviation for $1+\frac{c^{2}}{(k s \pm 2 a)^{2}}$.

Now one portion of this we know (cf. $\$ 10$ ), $\left\{\left(1+\frac{c^{2}}{2^{2} s^{2}}\right)\left(1+\frac{c^{2}}{4^{2} s^{2}}\right)\left(1+\frac{c^{2}}{6^{2} s^{2}}\right) \ldots\right\}^{2}=\left(\frac{2 s}{\pi c} \sinh \frac{\pi c}{2 s}\right)^{2}=A^{2}$ say; and as $\mathrm{A}^{2} \frac{c^{2}}{\rho^{2}}$ equals the square of the rational part of the quantity inside the brackets of (12), it follows that the square of the irrational part is equal to the remaining factors of the above product. It may be written

$$
\begin{gathered}
\frac{\cosh \frac{2 c \pi}{s}-\cos \frac{2 a \pi}{s}}{1-\cos \frac{2 a \pi}{s}}=1+\frac{\sinh ^{2} \frac{c \pi}{s}}{\sin ^{2} \frac{a \pi}{s}}=\left(1+\frac{c^{2}}{a^{2}}\right)\left(1+\frac{c^{2}}{(s-a)^{2}}\right) \\
\left(1+\frac{c^{2}}{(s+a)^{2}}\right)\left(1+\frac{c^{2}}{(2 s-a)^{2}}\right)\left(1+\frac{c^{2}}{(2 s+a)^{2}}\right) \ldots,
\end{gathered}
$$

merely putting $s$ instead of $2 s$, and $a$ instead of $2 a$. 
Whether this result is otherwise obvious or not, it will serve as an example of how new products might sometimes be evaluated from resistance-expressions.

General resistance of a circular sector.

$\S 18$. The resistance of a circular sector containing two poles $A$ and $B$ will be twice the resistance of a wedge with the same angle, but in which, besides the poles $A$ and $B$, there also exist poles $A^{\prime}$ and $B^{\prime}$ at the points inverse to $A$ and $B$ with regard to the circle of which the sector is a part; that is, calling $\mathrm{R}$ the radius of the sector, $O \mathrm{~A}^{\prime}=\frac{\mathrm{R}^{2}}{r_{1}}, O \mathrm{~B}^{\prime}=\frac{\mathrm{R}^{2}}{r_{2}}$. The expression for the resistance will be (5) plus an additional expression

$\Re=(5)+\frac{1}{2 \pi \kappa \delta} \log$

$\left\{\left.\frac{\mathrm{R}^{2}}{r_{1} r_{2}}\right|^{n}+\left.\frac{r_{1} r_{2}}{\mathrm{R}^{2}}\right|^{n}-2 \cos \overline{n \overline{\alpha-\beta}}\right\}\left\{\left.\frac{\mathrm{R}^{2}}{r_{r_{1}} r_{2}}\right|^{n}+\left.\frac{r_{1} r_{2} r_{2}}{\mathrm{R}^{2}}\right|^{n}-2 \cos n \overline{\alpha+\beta}\right\}$

$\left(\left.\frac{\mathrm{R}}{r_{1}}\right|^{n}-\left.\frac{r_{1}}{\overline{\mathrm{R}}}\right|^{n}\right)\left(\left.\frac{\mathrm{R}}{r_{2}}\right|^{n}-\left.\overline{r_{2}} \overline{\mathrm{R}}\right|^{n}\right)\left(\left.\frac{\overline{\mathrm{R}}}{r_{1}}\right|^{2 n}+\left.\overline{r_{1}}\right|^{2 n}-2 \cos 2 n o\right)^{\frac{1}{2}}\left(\left.\overline{\mathrm{R}}\right|^{2 n}+\left.\overline{r_{2}}\right|^{2 n}-2 \cos 2 n \beta\right)^{\frac{1}{2}}$

$\S 19$. When the angle of the sector vanishes by $R$ becoming infinite, we get the case of a strip bounded towards one end, or what may be called an isosceles triangle whose equal angles are $\frac{\pi}{2}$. The pole $A$ being at a distance $c_{1}$ from the base and $a$ from one of the sides, while $B$ is at a distance $b$ from the same side, $c_{2}$ from the base, the above expression reduces to

$$
\begin{gathered}
\mathrm{R}=(11)+\frac{1}{2 \pi \kappa \delta} \log \\
\left(\cosh \frac{c_{1}+c_{2}}{s} \pi-\cos \frac{a-b}{s} \pi\right)\left(\cosh \frac{c_{1}+c_{2}}{s} \pi-\cos \frac{a+b}{s} \pi\right)
\end{gathered}
$$

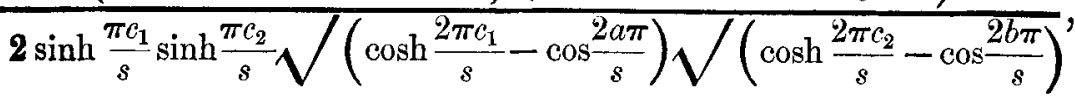

where the $c$ in (11) may be written $c_{1}-c_{2}$ for symmetry.

It may be noticed that the additional quantity in (14) vanishes when $c_{1}$ and $c_{2}$ are infinite; but becomes the same as (11) when $c_{1}$ and $c_{2}$ are made small and equal to $\frac{\rho}{2}$; and this is just what it ought to do.

$\S 20$. The quantity which is added to (11) to give (14) may be called the "effect of the boundary" which limits the strip at one end. If we added a boundary to the other end of the 
strip also, it would become a rectangle of breadth $s$ and length $c_{1}+c_{3}$ or $c_{2}+c_{4}$; and by adding another quantity to (11) similar to the above, but with $c_{3}$ and $c_{4}$. instead of $c_{1}$ and $c_{2}$ (that is, by adding a quantity which approximates to the effect of the new boundary), we should obtain an approximation to the resistance of a rectangle, which is a pretty close one if the $c$ are larger than the $s$. Such an approximate expression, however, has no particular interest. It is easy to write down the products occurring in ( $\alpha$ ) for the general case of a rectangle; but their evaluation does not appear to be so simple.

\section{Distribution of potential in a "wedge" containing any number of point poles.}

$\S 21$. All the flow-conditions in a wedge whose angle is $\frac{\pi}{n}$ are determined completely by writing out the particular form assumed by the general expression $(\mathrm{A})^{\prime}, \S 8$, for this case. The polar coordinates of the sources being $\left(r_{1}, \alpha\right),\left(r_{1}^{\prime}, \alpha^{\prime}\right) \ldots$, and of the sinks being $\left(r_{2}, \beta\right),\left(r_{2}^{\prime}, \beta^{\prime}\right) \ldots$, the potential at any point $(r, \phi)$ is

$$
\begin{aligned}
& \mathrm{V}=\frac{q}{4 \pi \kappa \delta} \log \\
& \frac{\left(r^{2 n}-2 r^{n} r_{2}^{n} \cos n \overline{\phi-\beta}+r_{2}^{2 n}\right)\left(r^{2 n}-2 r^{n} r_{2}^{n} \cos n \overline{\phi+\beta}+r_{2}^{2 n}\right)}{\left(r^{2 n}-2 r^{n} r_{1}^{n} \cos n \overline{\phi-\alpha}+r_{1}^{2 n}\right)\left(r^{2 n}-2 r^{n} r_{1}^{n} \cos n \overline{\phi+\alpha}+r_{1}^{2 n}\right)}
\end{aligned}
$$

+ similar terms with accented coordinates for every other pair of poles which the sheet may contain.

When the wedge becomes a "strip" of breadth $s$, the above expression gives, as the potential of any point $(x, \bar{y})$,

$$
\begin{aligned}
& \mathrm{V}=\frac{q}{4 \pi \kappa \delta} \log \\
& \frac{\left(\cosh \frac{y-y_{2}}{s} \pi-\cos \frac{x-x_{2}}{s} \pi\right)\left(\cosh \frac{y-y_{2}}{s} \pi-\cos \frac{x+x_{2}}{s} \pi\right)}{\left(\cosh \frac{y-y_{1}}{s} \pi-\cos \frac{x-x_{1}}{s} \pi\right)\left(\cosh \frac{y-y_{1}}{s} \pi-\cos \frac{x+x_{1}}{s} \pi\right)} \\
& +\& c .,
\end{aligned}
$$

where the sources are $\left(x_{1}, y_{1}\right),\left(x_{1}^{\prime}, y_{1}^{\prime}\right) \ldots$, the $\operatorname{sinks}\left(x_{2}, y_{2}\right)$, $\left(x_{2}^{\prime}, y_{2}^{\prime}\right) \ldots$, and where one side side of the strip is taken as the axis of $y$.

[To be continued.] 